\title{
Implicações da poluição veicular em praticantes de exercícios físicos em parque na cidade de Presidente Prudente - SP
}

A poluição veicular pode interferir na prática de exercícios físicos e na qualidade de vida das pessoas no ambiente urbano. O Parque do Povo, em Presidente Prudente - SP, apesar de ser um local com boa infraestrutura que proporciona a prática de exercícios físicos, é também um grande concentrador de tráfego de veículos. Desta forma, este trabalho teve como objetivo analisar a influência das possíveis implicações da poluição veicular em indivíduos praticantes de exercícios físicos regulares no Parque do Povo, na cidade de Presidente Prudente - SP. A hipótes adotada foi que a prática de exercício físico regular em parques urbanos pode interferir na qualidade respiratória do praticante, mesmo em ambientes abertos. $O$ estudo traz implicações e enfoques multidisciplinares, que se complementam dentro da perspectiva da saúde ambiental. A metodologia utilizada neste trabalho baseou-se na investigação qualitativa, utilizando-se das pesquisas de levantamento bibliográfico, documental, e de campo, cuja técnica contou com observação direta intensiva. O cálculo amostral foi baseado no estudo de Miot (2011), totalizando um número de 36 indivíduos. Os critérios de inclusão foram participantes possuírem idade entre 18 e 40 anos, praticarem exercícios físicos pelo menos três vezes na semana por, no mínimo, 50 minutos no Parque do Povo, não apresentar histórico de doenças pulmonares crônicas e/ou episódios recentes de infeç̃es nas vias áreas superiores. 0 estudo foi do tipo primá primánic do sintomas restio Stat. O estudo foi do tipo primário, de caráter longitudinal não controlado e prospectivo. O presente estudo mostrou que os participantes da pesquisa se tornaram mais ativos, sendo possivelmente estimulados e motivados pelo ambiente com grande presença de vegetação e a boa infraestrutura do local e pelas orientações realizadas durante a pesquisa.

\section{Implications of vehicular pollution in practicing regular physical exercise in open-air Park in the city of Presidente Prudente - SP}

\begin{abstract}
The vehicular pollution may interfere with the practice of physical exercise and the quality of life of people in the urban environment. The People's Park in Presidente Prudente - SP, despite being a place with good infrastructure that provides physical exercise, is also a major hub of vehicular traffic. With regular practice of physical exercises we present benefits to the population as physical, social and affective health. In this way, this work aims to analyze the influence of the possible implications of vehicular pollution on individuals practicing regular physical exercises in the People's Park in the city of Presidente Prudente-SP. The questions that led to the accomplishment of this work are that the pollution can cause respiratory problems in the practitioners of physical exercise, and also, taking into account that we have the climate as an important variable and the best time to do physical exercise in the place presented. The hypothesis adopted is that the practitioner of physical exercise in the open environment with the presence of environmental pollution on some implication of their well being. The methodology used was the bibliographical research in selected literature and applied research with practitioners of physical activities in the People's Park, information was collected through interviews, systematic observation, an evasion pump to capture carbon monoxide present in the air of the place The determination of air pollutants will be performed through the automatic monitoring network to ether with the daily values of temperature, relative humidity, precipitation, direction and wind speed and Coex for the measurement of carbon monoxide in exhaled air of participants toget before and after physica exercise, therefore presented the air pollution in specific vehicular pollution and its effects on the environment and human healt, following, demonstrated and contextualized the demographic characteristics of the city and of the people's park, and above all that the place studied has great importance for city and regional development, valuing the environment and the accomplishment of physical exercise and well-being measured to the population. The results showed individuals with hemodynamic factors (PAS, PAD, FC, FR) within the normal established standard, more active presented in the IPAQ, being able to relate that the participants were stimulated and motivated by the extensive vegetation in the studied place.

Keywords: Physical Exercise; Air Pollution; Urban Green Areas; Health.
\end{abstract}

Topic: Epidemiologia e Saúde Ambiental

Reviewed anonymously in the process of blind peer.

Alba Regina Azevedo Arana (D)

Universidade de São Paulo, Brasil

http://lattes.cnpq.br/1931029698985787

http://orcid.org/0000-0001-8995-4449

alba@unoeste.br

Juliana Felipe

Universidade do Oeste Paulista, Brasil http://lattes.cnpq.br/4911289356617734

jedfisica@gmail.com

Marcus Vinicius Pimenta Rodrigues

Faculdade de Medicina de Botucatu, Brasil

http://lattes.cnpq.br/0133739307461180

marcusvinicius@unoeste.br

d

DOI: 10.6008/SPC2179-6858.2017.004.0004
Received: 05/07/2017

Approved: 05/10/2017

\author{
Aline Duarte Ferreira \\ Universidade Estadual Paulista Júlio de Mesquita Filho, Brasil \\ http://lattes.cnpq.br/8550928028795394 \\ aline@unoeste.br \\ Victor Cesar Belloni dos Santos \\ Universidade do Oeste Paulista, Brasil \\ http://lattes.cnpq.br/3909977946361423 \\ victorcesar.belloni@hotmail.com \\ Gabrielle Gomes Ribeiro \\ Universidade Estadual Paulista Júlio de Mesquita Filho, Brasil \\ http://lattes.cnpq.br/8506686504401571 \\ gabrielle@unoeste.br
}

Referencing this:

ARANA, A. R. A.; FELIPE, J.; RODRIGUES, M. V. P.; FERREIRA, A. D. SANTOS, V. C. B. RIBEIRO, G. G.. Implicações da poluição veicular em praticantes de exercícios físicos em parque na cidade de Presidente Prudente - SP. Revista Ibero-Americana de Ciências Ambientais, v.8, n.4, p.37-54, 2017. DOI: http://doi.org/10.6008/SPC2179-

6858.2017.004.0004 


\section{INTRODUÇÃO}

Atualmente, a população apresenta cada vez mais consciência de que a prática de exercício físico é necessária não só para aqueles que desejam manter a boa aparência, como também para manutenção da saúde, envolvendo todos os indivíduos, de todas as classes sociais e faixas etárias. Os malefícios do sedentarismo superam significativamente as eventuais complicações decorrentes da prática dos exercícios físicos, os quais, portanto, apresentam uma interessante relação de benefício e risco, por isso a importância do local onde será praticado o exercício físico, em qual ambiente essa atividade será realizada (MIRANDA; BATISTA, 2008).

Ambientes como as áreas verdes possibilitam o combate à poluição atmosférica por meio da fotossíntese, além de regular a umidade e a temperatura do ar e ainda reduzir os níveis de ruído, servindo como amortecedor do barulho das cidades (BENINI, 2009). A exposição à poluição atmosférica é uma das grandes causadoras de doenças respiratórias crônicas, influenciando a exacerbação da doença e diminuição da função pulmonar (LEBOWITZ, 1996, citado por LASTE, 2009). Este assunto é de interesse global e merece atenção dos profissionais de saúde, dos ambientalistas, dos gestores públicos, uma vez que a questão envolve políticas públicas destinadas a promover a saúde, a prevenir as doenças e a proporcionar melhores condições de vida para as pessoas.

Esta pesquisa tem implicações e enfoques multidisciplinares, que se complementam dentro da perspectiva da saúde ambiental. A vida urbana nas cidades é vista, muitas vezes, como estressante, poluída e com diversos conflitos que podem afetar a qualidade de vida das pessoas. Desta forma, os parques urbanos com área verde contribuem para melhoria da qualidade de vida, bem como para a saúde e o bem-estar da população.

Destarte, este artigo tem como objetivo abordar a prática do exercício físico em um parque a céu aberto, suscitando as possíveis relações da exposição à poluição veicular ao redor do parque com a presença de sintomas respiratórios. E ainda discutir a influência da poluição atmosférica na saúde da população urbana; caracterizar a população estudada por meio das características antropométricas, análise do nível de atividade física e percepção subjetiva do esforço físico e avaliar os sintomas respiratórios e mensurar a quantidade de monóxido de carbono no ar exalado (COex), a fim de verificar a influência da poluição atmosférica nos indivíduos estudados.

\section{METODOLOGIA}

A metodologia utilizada foi baseada na investigação qualitativa, utilizando-se da pesquisa de levantamento bibliográfico, da pesquisa documental e da observação direta intensiva. A abordagem qualitativa visa relacionar a teoria e os dados, a teoria e a ação, por meio da compreensão dos fenômenos a partir da descrição e interpretação, bem como das experiências pessoais do pesquisador (TEIXEIRA, 2000).

A metodologia empregada recaiu na pesquisa bibliográfica em literaturas selecionadas e a pesquisa de campo se deu com praticantes de exercícios físicos no Parque do Povo em Presidente Prudente/SP. A 
pesquisa de campo aconteceu em dois momentos: primeiro momento no mês de fevereiro/2016 e segundo momento em julho/2016, sempre entre o período das $17 \mathrm{~h}$ às $19 \mathrm{~h}$.

O cálculo amostral foi baseado no estudo de Miot (2011), totalizando um número de 36 indivíduos. Os critérios de inclusão foram participantes possuírem idade entre 18 e 40 anos, praticarem exercícios físicos pelos menos três vezes na semana por no mínimo 50 minutos, no Parque do Povo, não apresentar histórico de doenças pulmonares crônicas e/ou episódios recentes de infeções nas vias áreas superiores. A participação dos sujeitos foi iniciada apenas após aprovação do estudo no Comitê de Ética e Pesquisa (CEP) da Universidade do Oeste Paulista (CAAE 46057715.2.0000.5515).

O estudo foi do tipo primário, de caráter longitudinal não controlado e prospectivo. Os participantes passaram por uma avaliação inicial, para obtenção de dados de identificação pessoal, sinais vitais morbidades preexistentes, investigação de histórico tabagístico e dados antropométricos. Na sequência, realizaram a mensuração do monóxido de carbono no ar exalado (COex) e responderam a dois questionários validados na língua portuguesa: o de sintomas respiratórios e o IPAC- International Physical Activity Questionnarie validado por Pardini et al. (2001). A percepção de esforço foi avaliada por meio da escala de Borg e a determinação dos poluentes atmosféricos foi realizada por meio da rede de monitoramento automática, juntamente com os valores diários de temperatura, umidade relativa do ar e velocidade.

Após esse primeiro período de avaliações, os participantes forma convidados a realizar uma caminhada de intensidade moderada por 30 minutos por um percurso preestabelecido no Parque do Povo, cerca de $2 \mathrm{~km}$, conforme se nota na figura 1 . A caminhada sempre ocorreu de forma supervisionada e a percepção do esforço físico foi verificada por meio da escala de Borg. Após a caminhada, os participantes foram reavaliados, e as medidas de COex e sinais vitais foram repetidas imediatamente após a caminhada e após 15 minutos de repouso (volta à calma).

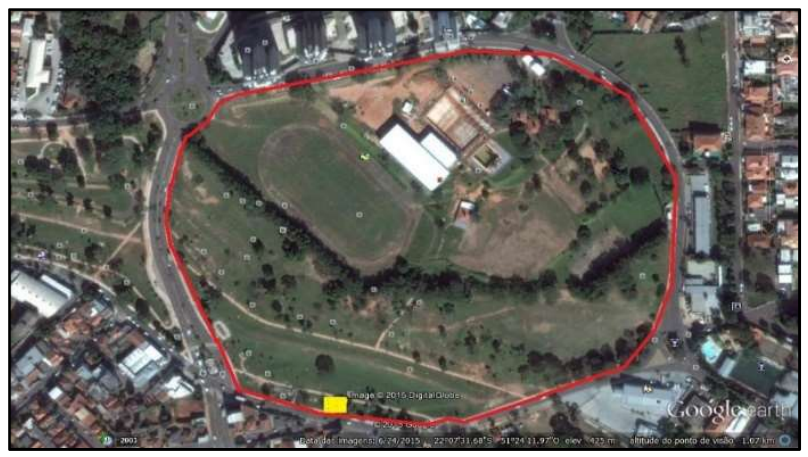

Figura 1: Trajeto Parque do Povo.

Para avaliação dos sintomas respiratórios, utilizamos um questionário da European Community Respiratory Health Survey (BIINO, 2001) validado para a língua portuguesa (RIBEIRO, 2007). O instrumento é constituído de oito questões de fácil interpretação, e rápida aplicação. As questões são referentes às queixas respiratórias, assim como alergias, espirros frequentes, coceiras no nariz entre outros. O questionário foi aplicado como entrevista, sem induzir o entrevistado às respostas.

Todas as análises estatísticas foram feitas por intermédio do software Action Stat, um sistema estatístico que usa a linguagem $R$ e trabalha de forma integrada ao Excel, utilizando uma interface fácil e 
amigável. Primeiramente, realizamos uma análise descritiva dos dados, por meio do cálculo de medidas como média e desvio-padrão e construção de tabelas e gráficos de barras. Depois, dividiu-se o conjunto de dados em grupos como: primeira e segunda fase; sexo Feminino e sexo Masculino. Esses grupos foram comparados em relação a algumas variáveis do conjunto de dados.

\section{Impactos ambientais e a sua relação com a poluição atmosférica}

Todo impacto ambiental tem uma ou mais causas, e se constitui do resultado das ações humanas sobre os aspectos ambientais. A causa do impacto ambiental, muitas vezes, tem relação direta e indireta com a poluição ambiental. A definição de poluição ambiental é muito semelhante à definição de impacto ambiental, no entanto, um impacto ambiental pode ser negativo ou positivo, ou seja, ele pode tanto trazer prejuízos como benefícios (CONAMA, 1986).

Mota (2003) entende impacto ambiental como a cadeia de efeitos que se produzem no meio natural e social (antrópico), como consequência de uma determinada ação. É muito importante interpretarmos o item sobre a poluição que pode ser causada por empreendimentos que disponham no meio ambiente efluentes, emissões, resíduos ou energia acima dos padrões ambientais, isto é, valores limites estabelecidos.

Os desequilíbrios ambientais que se traduzem na deterioração ambiental nas mais diversas regiões do planeta, e que comprometem a qualidade do ar, água e solo, além da qualidade de vida e sobrevivência humana, obrigaram as sociedades a intensificarem movimentos ambientalistas, contribuindo para a difusão do interesse da conservação e preservação ambiental em escala global (PELICIONI, 2005).

Os impactos ambientais podem ser diretos ou indiretos; produzir-se a curto ou a longo prazo; ser de curta ou longa duração; ser cumulativos; reversíveis ou não; ser inevitáveis; locais, regionais, continentais, globais; naturais e antropogênicos (BRILHANTE et al., 1999). A poluição atmosférica é um grave impacto ambiental das grandes cidades no Brasil.

Os parâmetros indicadores da qualidade do ar apontados nesta Resolução correspondem às partículas totais em suspensão (PTS), fumaça, partículas inaláveis (PI), dióxido de enxofre $\left(\mathrm{SO}_{2}\right)$, monóxido de carbono (CO), ozônio $\left(\mathrm{O}_{3}\right)$ e dióxido de nitrogênio $\left(\mathrm{NO}_{2}\right)$. Para a avaliação e fixação dos limites de concentração que assegurem a saúde e o bem-estar das pessoas, foram definidos dois padrões de qualidade do ar: os padrões primários, os quais ultrapassados “[...] poderão afetar à saúde da população, podendo ser entendidos como níveis máximos toleráveis de concentração de poluentes atmosféricos, constituindo-se em metas de curto e médio prazo" (CONAMA, 1986). Pode-se dizer que os padrões primários se constituem no limite inferior das concentrações a partir do qual o contaminante pode causar prejuízos à população e ao seu meio. Os padrões secundários correspondem às concentrações de contaminantes atmosféricos abaixo das quais se prevê o mínimo efeito adverso sobre o bem-estar da população, assim como o mínimo dano à fauna, à flora, aos materiais e ao meio ambiente em geral (CONAMA, 1986).

A determinação sistemática da qualidade do ar deve ser, por questões de ordem prática, limitada a um restrito número de poluentes, definidos em razão de sua importância e dos recursos materiais e humanos disponíveis. De uma forma geral, a escolha recai sempre sobre um grupo de poluentes que servem como 
indicadores de qualidade do ar, consagrados universalmente, a saber: dióxido de enxofre $\left(\mathrm{SO}_{2}\right)$, material particulado (MP), monóxido de carbono $(\mathrm{CO})$, ozônio $\left(\mathrm{O}_{3}\right)$ e dióxido de nitrogênio $\left(\mathrm{NO}_{2}\right)$. A razão da escolha desses parâmetros como indicadores de qualidade do ar está ligada à sua maior frequência de ocorrência e aos efeitos adversos que causam ao meio ambiente (CETESB, 2014).

Especificamente sobre o sistema respiratório, as vias aéreas estão constantemente expostas aos microrganismos provenientes da inalação de poluentes e gases tóxicos nocivos. Entre os poluentes atmosféricos, a exposição ao $\mathrm{NO}_{2}, \mathrm{O}_{3}$, e MP tem sido associada ao aumento na mortalidade da população e na admissão hospitalar por doenças respiratórias, tanto em estudos que avaliam efeitos agudos como crônicos na saúde humana (SALICIO, 2016).

O estudo de Riechelmann et al. (2003) deixou evidente que a exposição aguda por um período de três horas ao MP em concentrações ambientais pode provocar aumento no transporte mucociliar, diminuição do fluxo de ar na cavidade nasal e aumento da sensação de obstrução nasal em indivíduos saudáveis, prejudicando, dessa maneira, as funções desempenhadas pelo nariz.

Segundo Braga (2003), a poluição tem causado alterações clínicas na população, como sintomas respiratórios e cardiovasculares, crises de asma, limitação funcional, aumento no número de consultas em pronto-socorro e internações hospitalares. Há evidências de que a inalação aguda do MP desencadeia desconforto respiratório, crises de espirro, dores de cabeça e ardência nos olhos em indivíduos saudáveis (FISHMAN et al., 2008). Nos indivíduos vulneráveis (doentes crônicos, idosos e crianças asmáticas) ocorre o agravamento dos sintomas e da doença.

As principais fontes emissoras de CO são os veículos automotivos, aquecedores a óleo, queima de tabaco, churrasqueiras e fogões a gás (CANÇADO, 2006). O CO é o poluente mais comum no ar das grandes cidades, uma vez que sua fonte principal são as emissões de automóveis. Assim, a exposição ao CO é particularmente alta perto de vias com muito tráfego (MIRANDA et al., 2008).

Logo, a prática de exercício físico próximo a vias de trânsito (ruas, avenidas, estradas) implica grandes inalações de CO. A exposição ao CO pode causar dor de cabeça, fadiga e sintomas iguais ao da gripe, efeitos cardíacos diversos que incluem a diminuição da capacidade de se exercitar (CASTRO, et al., 2003).

O principal efeito do CO na saúde está associado à capacidade de transporte de $\mathrm{O}_{2}$ pela hemoglobina. A hemoglobina, também chamada de hemácia ou eritrócito, combina-se com $\mathrm{O}_{2}$ com uma afinidade 200 vezes maior do que se combina com o $\mathrm{O}_{2}$. Ao formar a carboxihemoglobina, composto resultante da reação da hemoglobina com o $\mathrm{CO}$, a possibilidade de $\mathrm{O}_{2} \mathrm{O}_{2}$ ser transportado pela hemoglobina às células do organismo é reduzida. O CO, após se combinar com a hemoglobina, exerce efeito tóxico nos capilares pulmonares. Portanto, nos pulmões a hemoglobina troca $\mathrm{CO}_{2}$ por $\mathrm{O}_{2}$ e nos tecidos a troca é inversa, $\mathrm{O}_{2}$ por $\mathrm{CO}_{2}$ (LASTE, 2009).

O monóxido de carbono entra com facilidade nos pulmões e no sangue, combinando-se com a hemoglobina e dificultando o transporte de oxigênio para os tecidos, podendo, assim, causar dois tipos de intoxicação no corpo humano. A intoxicação crônica - cujos sintomas são dores de cabeça, náuseas, vômitos e cansaço - poderá se desenvolver de forma lenta e afetar pessoas habitualmente expostas a concentrações 
elevadas de CO. E a intoxicação aguda pode provocar vertigens, fraqueza muscular, distúrbios visuais, taquicardia, perturbações de comportamento, desmaios e, no limite, o coma e mesmo a morte (LASTE, 2009).

Esteves (2004) observou, em seu estudo, que pessoas saudáveis e não fumantes residentes em áreas com altos índices de CO apresentaram aumento de até 100\% nos níveis de carboxihemoglobina quando comparadas com pessoas saudáveis e não fumantes que não estão expostas a altos índices de CO no trânsito urbano. É comumente associado a intoxicações, focando seus efeitos principalmente sobre o coração e pulmão.

Por causa do ozônio, a função pulmonar é variavelmente debilitada e a capacidade pulmonar pode diminuir. Os efeitos do $\mathrm{O}_{3}$ são maiores durante a realização de exercícios físicos, pois ocorre um aumento da suscetibilidade dos pulmões, quanto a infecções, alergias e, inclusive, há influência de outros contaminantes. $\mathrm{O}$ ser humano exposto a altas concentrações de $\mathrm{O}_{3}$ pode apresentar tosse, chiado na respiração e uma dor no peito peculiar na região subesternal, comumente arrebatadora ou de caráter de queimação, que aumenta gradualmente em intensidade na inspiração e declina na expiração (DICKEY, 2000). Outros estudos relatam que os efeitos dos poluentes atmosféricos, tanto a curto quanto a longo prazo, causam também efeitos adversos sobre a função pulmonar (CANÇADO, 2006).

Ainda nesse sentido, outro estudo observou que os poluentes atmosféricos ocasionam aumento da incidência de infecções do trato respiratório e sintomas respiratórios, levando à piora da função pulmonar e ao agravamento de doenças crônicas preexistentes (ARBEX et al., 2012). Sabe-se que o material particulado é o maior componente da poluição atmosférica, e que ele exerce um efeito negativo sobre a saúde humana. Os efeitos adversos do MP sobre o epitélio respiratório de seres vivos podem manifestar-se de forma aguda - horas ou dias após a exposição - ou de forma crônica - após longos períodos de exposição (OLIVEIRA, 2006). A deposição deste poluente no trato respiratório está relacionada com o tamanho das partículas e da eficiência dos mecanismos de defesa das vias aéreas, conforme representado na figura 2.

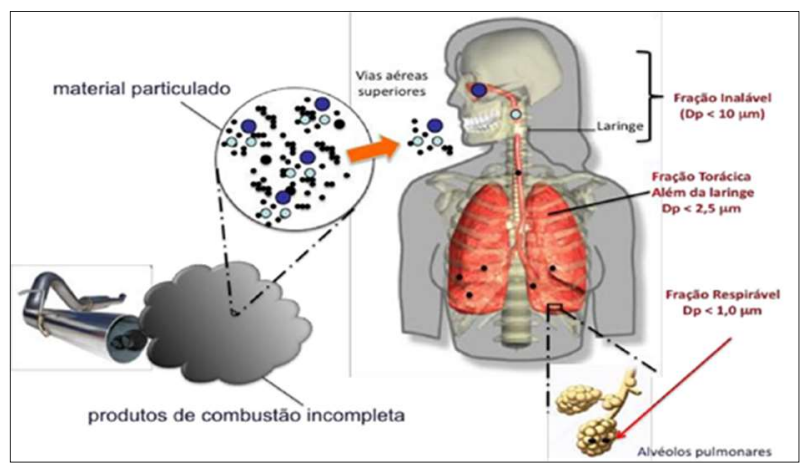

Figura 2: Diferentes diâmetros de partículas do material particulado e sua deposição no trato respiratório.

A industrialização generalizada do século XX, impulsionada pela eletricidade e motor a combustão, conduziu não só a sua diversificação, como também a sua disseminação em escala mundial (OLIVERA, 2007). São vários e alarmantes os episódios críticos de poluição com perdas de vidas ou danos à saúde, que passaram a ser registrados em meados deste século, em decorrência de emissões de contaminantes por diferentes tipos de indústrias nas mais variadas regiões do globo, associados a condições meteorológicas de fraca dispersão de poluentes, como alguns exemplos que mencionamos a seguir. 
As características dos poluentes do ar variam e suas concentrações mudam espacialmente e temporalmente. Embora todos sejam suscetíveis a alta concentração de poluição, suas concentrações não são distribuídas uniformemente entre as populações. Em razão de tais complexidades, ainda existem muitas questões de pesquisa a serem abordadas por futuros estudos epidemiológicos sobre a poluição do ar (BRAGA, 2003).

O balanço do Denatran aponta que o Brasil fechou, em 2010, com exatos 64.817 .974 veículos registrados. Em dez anos, o aumento acumulado é de 119\%, ou seja, mais 35 milhões de veículos chegaram às ruas no período. Segundo o órgão, essa seria a frota circulante no país e considera carros, motos, caminhões e outros tipos de automotores inseridos no cadastro desde 1990 (DENATRAN, 2014).

Entretanto, apresentamos alguns dados sobre tal afirmação, como por exemplo, o recorde de vendas de carros traz apagão de autopeças, o financiamento de veículos bate recorde e encerra em $\mathrm{R} \$ 188,6 \mathrm{bi}$, a inspeção veicular ambiental em SP vai ficar mais cara (DENATRAN, 2014). Considerando o resultado do Censo IBGE (2010), que indica que a população é de 190,732mi, o país tem uma média de um carro para cada 2,94 habitantes.

Cabe ainda apontarmos algumas considerações diante dos avanços obtidos no controle de qualidade dos combustíveis e das emissões veiculares no Brasil. Proposto pela CETESB, o Programa de Controle de Poluição do Ar por Veículos Automotores (PROCONVE), após dois anos de estudos para sua viabilização, foi adotado nacionalmente via CONAMA, e regulamentado pela Resolução no 18 de 6/5/1986 do mesmo órgão. Entre os objetivos do Programa, destacamos a redução das emissões de poluentes veiculares visando atingir os padrões de qualidade do ar, bem como a melhoria das características técnicas dos combustíveis utilizados pelos veículos automotores (CONAMA, 1986).

Observamos que, em Presidente Prudente, a relação habitantes/veículos sofreu um decréscimo anual de 7,02\%, ao passo que na capital do Estado, o decréscimo foi de apenas 4,47\%, 57\% menor, fato este que não é favorável ao município, visto que, quanto menor esta relação maior o número de veículos por habitantes, e, consequentemente, mais problemas nas vias da cidade (DENATRAN, 2014). Apesar de complexo, o problema pode ser minimizado por medidas ao alcance da sociedade. Para isso, é indiscutivelmente importante o trabalho multidisciplinar, aplicando medidas para reduzir a poluição atmosférica, sendo este um assunto tratado como questão de saúde pública.

\section{Parque do Povo da cidade de Presidente Prudente}

O Parque do Povo localiza-se na porção sudoeste da cidade de Presidente Prudente, como pode se visualizar na figura 3 , constitui um faixa linear, de cerca de $3 \mathrm{~km}$ de extensão que possui em seu interior, além de grandes gramados e bom número de árvores, áreas de múltiplo uso, que incluem pistas e quadras para práticas desportivas, lanchonetes, postos policiais e até serviço de fotocópias.

Em seu entorno, altamente valorizado do ponto de vista do mercado imobiliário, existe um grande número de restaurantes, bares, academias de ginástica e lojas. É delimitado e cruzado pelas principais vias arteriais da cidade. Todos esses atrativos, aliados à localização estratégica, fazem do Parque do Povo uma 
área de lazer dentro do espaço urbano (SAWADA, 2007). O parque é intensamente frequentado para o uso de suas pistas de corrida, quadras esportivas, pistas de skate, gramados e calçadas que o circundam.

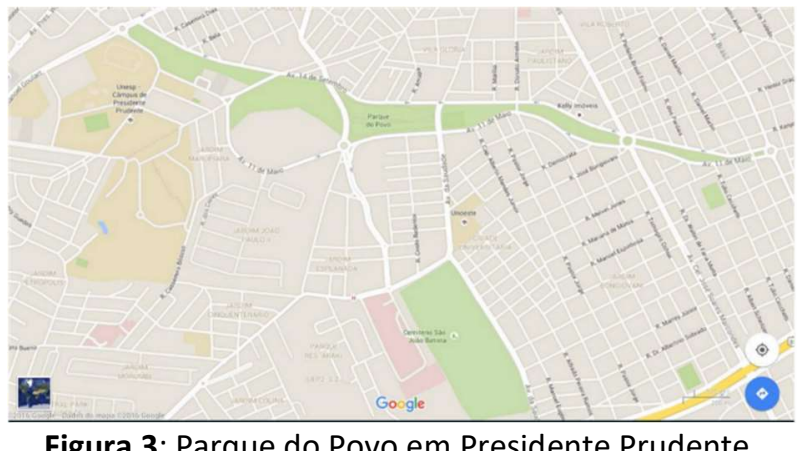

A Prefeitura Municipal de Presidente Prudente, no ano de 1976, apresentou à população da cidade um projeto denominado 'Fundo de Vale' (COSTA, 2009). O projeto consistia na implantação de um parque em uma região de fundo de vale por onde passa o Córrego do Veado, justificado pela necessidade de recuperação dessa área que era bastante degradada e constituía uma barreira física para a circulação entre a parte central da cidade e os bairros além dele localizados, em direção à rodovia Raposo Tavares (SAWADA, 2007).

Inicialmente, a obra consistiu na implantação de duas avenidas, atualmente 11 de Maio e 14 de Setembro, fazendo uma ligação entre a Avenida Brasil e a Avenida Manoel Goulart, ladeando o Córrego do Veado, em uma distância que possibilitaria a implantação de uma série de equipamentos de lazer para a população entre as duas avenidas. O projeto previa, também, a canalização do Córrego do Veado entre a Avenida Brasil e a Avenida Manoel Goulart, até se encontrar com o Córrego da Colônia Mineira (próximo ao Prudenshopping) (SILVA, 1994).

Parcialmente construído, o Parque do Povo foi inaugurado em 1982. Mas, um ano depois, foi abandonado pela administração municipal por constituir-se uma obra demasiadamente onerosa e por não ter solucionado o problema das inundações nessa área. Além disso, a ocorrência da queda das placas de proteção das margens do córrego agravou ainda mais a situação. De acordo com a Secretaria de Planejamento da Prefeitura, os projetos que financiaram essas obras iniciais endividaram a prefeitura até o ano de 2019 (VAZ, 1999).

Em 1995, a Prefeitura, com apoio da Companhia Prudentina de Desenvolvimento (PRUDENCO), iniciou as obras referentes à substituição da canalização do Córrego do Veado, no Parque do Povo, pela canalização fechada, constituída por tubos ármicos corrugados. As 14 obras foram executadas em etapas e tiverem custo estimado em cinco milhões de reais (SILVA, 1994). Em 1997, um desmoronamento de parte da Avenida Tancredo Neves ocasionou a paralisação das obras.

Conforme Sobarzo (2005, citado por COSTA, 2009), no ano de 2001 a Prefeitura Municipal lançou a 'campanha de revitalização do parque', pedindo à população sugestões por meio de um encarte distribuído por um jornal da cidade: "Esta campanha visa tornar mais bela a maior área urbana e de lazer do município. Vamos transformá-la num cartão postal. Participe!". 
Houve, então, em 2002, o reinício da revitalização do parque, com ampliação das calçadas, dos estacionamentos e do parque infantil e implantação dos campos de areias, pista para bicicletas e circuitos de exercícios. Em 2005, iniciaram-se as obras de finalização, que incluíram a instalação de um posto da Policia Militar para maior segurança dos frequentadores.

O Parque do Povo é uma área utilizada pela população prudentina em geral como área de lazer e para a prática de seus esportes. O lazer é hoje um elemento do processo de reprodução, um tempo que se organiza em virtude da reprodução das relações sociais e este tempo tem a mesma propriedade do espaço que organiza toda a vida social e que também organiza a sociedade de consumo, igualmente os ambientes de lazer (BORTOLO, 2013). O Parque deve ser vislumbrado como um espaço de possibilidades, como salienta Sobarzo (2005), cujas diversas ações são produzidas e consumidas neste local público. Indo do simples ato de realizar uma caminhada, como também de um espaço para a manifestação popular em um momento de realizações da sociedade político-social prudentina (figura 3).

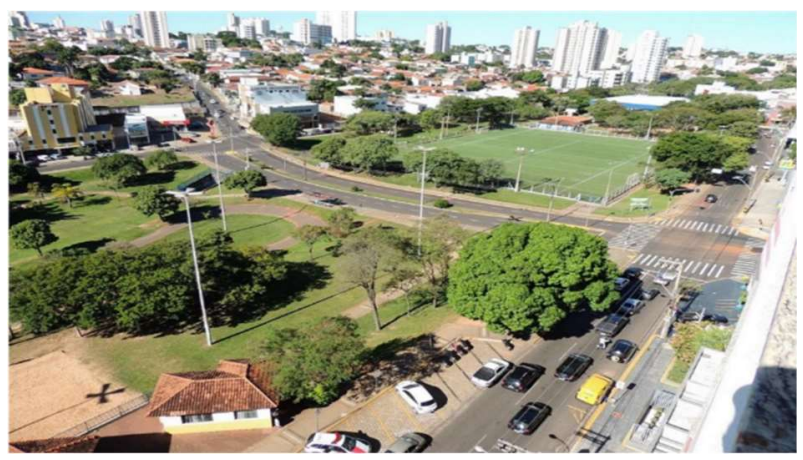

Figura 3: Parque do Povo.

Observado o grande fluxo de pessoas que diariamente utilizam as dependências desse parque, é importante analisar de que maneira esse espaço é utilizado e qual o papel que ele desempenha na autopercepção de saúde dos cidadãos que buscam o bem-estar por meio de uma vida fisicamente ativa. Reconhecendo a interdisciplinaridade dos estudos ambientais, é importante caracterizar o perfil dos atuais frequentadores do parque e identificar as possíveis implicações da poluição veicular destes indivíduos ao praticarem exercício físico em um parque a céu aberto.

\section{RESULTADOS E DISCUSSÃO}

Os resultados que apresentamos correspondem à amostra de 36 indivíduos (17 mulheres e 19 homens), acompanhados em duas fases: em janeiro/2016 (verão) e julho/2016 (inverno). A tabela 1 indica a caracterização dos participantes da pesquisa nos dois momentos. Esses valores foram captados sempre em repouso, antes do início da intervenção. Os valores encontram-se em médiat desvio-padrão. Na 10 fase (fev/2016), teve-se um total de 36 respondentes, sendo 17 mulheres e 19 homens. Na 20 fase (jul/2016), teve-se o mesmo número de respondentes. Como mostra a Tabela 1, a média da idade para homens foi de 27 anos, sendo $22 \%$ deles com idade de 18 a 23 e $36 \%$ com idade de 24 a 30 anos. A média de idade para as mulheres foi de 23 anos, sendo $27 \%$ com idades de 18 a 23 e $11 \%$ com idade de 24 a 30 anos. 
Tabela 1: Descrição da caracterização dos participantes nas duas fases: fev/2016 e jul/2016, respectivamente.

\begin{tabular}{|l|c|c|}
\hline Variáveis & \multicolumn{2}{|c|}{ Média e desvio-padrão } \\
\hline & HOMENS & $23,30 \pm 5,7$ \\
\hline Idade (anos) & $27,42 \pm 5,8$ & $1,65 \pm 0,0$ \\
\hline Altura (m) & $1,77 \pm 0,0$ & $64,40 \pm 17,2$ \\
\hline Peso (kg) & $82,78 \pm 17,5$ & $23,56 \pm 4,6$ \\
\hline IMC (kg/m) & $26,45 \pm 4,8$ & $74,38 \pm 14,3$ \\
\hline Circunferência abdominal & $87,63 \pm 14,4$ & $112,35 \pm 6,6$ \\
\hline PA (sistólica) (mmHg) & $119,79 \pm 8,2$ & $75,88 \pm 7,1$ \\
\hline PA (diastólica) (mmHg) & $77,89 \pm 17,8$ & $79,53 \pm 12,6$ \\
\hline Frequência cardíaca & $86,74 \pm 11,7$ & $25,12 \pm 2,9$ \\
\hline Frequência respiratória & $27,58 \pm 3,1$ & MULHERES \\
\hline Variável & \multicolumn{2}{|c|}{ Média e desvio-padrão } \\
\hline & HOMENS & $23,30 \pm 5,7$ \\
\hline Idade (anos) & $27,42 \pm 5,8$ & $1,65 \pm 0,0$ \\
\hline Altura (cm) & $1,77 \pm 0,0$ & $64,40 \pm 17,2$ \\
\hline Peso (kg) & $82,78 \pm 17,5$ & $42,91 \pm 27,2$ \\
\hline IMC (kg/m) & $46,98 \pm 32,7$ & $75,53 \pm 11,4$ \\
\hline Circunferência abdominal & $93,18 \pm 13,8$ & $110,00 \pm 8,8$ \\
\hline PA (sistólica) (mmHg) & $122,94 \pm 11,6$ & $74,37 \pm 6,1$ \\
\hline PA (diastólica) (mmHg) & $63,53 \pm 31,0$ & $77,84 \pm 7,7$ \\
\hline Frequência cardíaca & $80,71 \pm 12,8$ & $23,10 \pm 1,8$ \\
\hline Frequência respiratória & $23,23 \pm 3,1$ & \\
\hline
\end{tabular}

Legenda: IMC = índice de massa corpórea; $\mathrm{Kg}$ = quilograma; $\mathrm{m}$ = metro; $\mathrm{cm}$ = centímetro; PS=pressão arterial sistólica; PD=pressão arterial diastólica.

A seguir, tratamos dos valores de média \pm desvio - padrão de pressão arterial diferenciando os sexos. A média dos dados de pressão arterial sistólica na primeira fase (fev./2016) dos homens participantes foi de $119,7 \mathrm{mmHg}$ e das mulheres $112,3 \mathrm{mmHg}$, valores considerados dentro da normalidade. Na segunda fase (jul./2016) a média para os homens registrada foi de $122,9 \mathrm{mmHg}$, sendo maior que a primeira fase e acima do valor normal padrão.

Considera-se hipertensão arterial quando a pressão arterial sistólica se encontra maior ou igual a 140 $\mathrm{mmHg}$ e a pressão arterial diastólica maior ou igual a $90 \mathrm{mmHg}$, em indivíduos que não estão fazendo uso de medicação anti-hipertensiva. A pressão arterial normal estabelecida pela área médica é de $<120 / 80$ (ANDRADE, 2010). Desse modo, observamos que a pressão arterial de todos os participantes do presente estudo encontra-se normal.

A média dos dados de pressão arterial diastólica na primeira fase (fev./2016), para os homens foi de $77,8 \mathrm{mmHg}$ e para as mulheres foi de $75,8 \mathrm{mmHg}$, apresentando dados normais. Na segunda fase (jul./2016) a média das aferições dos participantes homens foi $63,5 \mathrm{mmHg}$ e a média das mulheres foi $74,3 \mathrm{mmHg}$, se mantendo nos padrões normais estabelecidos.

A média de frequência cardíaca na primeira fase (fev/2016) foi para homens $86,7 \mathrm{bpm}$ e para mulheres 79,5bpm, apresentando dados normais. Na segunda fase (jul/2016) a média das aferições para homens foi de $80,7 \mathrm{bpm}$ e a média das mulheres foi de $77,8 \mathrm{bpm}$, se mantendo nos padrões normais estabelecidos. Cabe salientarmos que os valores que identificamos junto aos participantes desta pesquisa estão de acordo com Teixeira (2000), quando assevera que exercício físico de baixa intensidade diminui a pressão arterial porque provoca redução no débito cardíaco, o que pode ser explicado pela diminuição na frequência cardíaca de repouso e diminuição dos tônus simpáticos no coração, em decorrência de menor intensificação simpática e maior retirada vagal. 
Em outro estudo, autores mostram que indivíduos bem treinados ou bem condicionados fisicamente aerobiamente possuem frequência cardíaca de repouso mais baixa, sugerindo maior atividade parassimpática, ou menor atividade simpática, como explicação fisiológica para esse fato (AUBERT et al., 2001). Com relação ao IMC e à frequência respiratória dos participantes da primeira fase, notamos uma correlação, pois, as variáveis aumentaram respectivamente, mas pelo valor foi considerada fraca ( $p$-valor 0,48 ) e na segunda fase, não registramos uma correlação significativa ( $p$-valor 0,08 ).

Os entrevistados ainda foram avaliados quanto à percepção esforço. A escala do Borg, proposta no gráfico 1, é um instrumento que avaliou a intensidade do exercício em termos de determinações em índices subjetivos, conforme a percepção do indivíduo, proporcionando diretamente uma medida individualizada da percepção da dispneia e da fadiga no exercício.

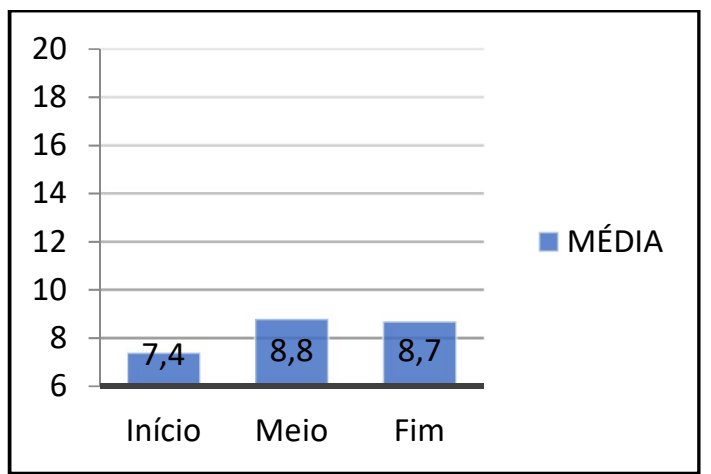

Gráfico 1: Descrição de média da escala de Borg dos participantes.

Durante a prática de exercício físico, a frequência cardíaca sofre alterações, portanto, usamos a escala de Borg como padrão subjetivo de esforço físico, utilizada para qualquer exercício aeróbio, sendo recomendada como uma opção prática na observação. O Gráfico 1 indica a média da escala de Borg dos participantes de 7,4 no início do percurso, classificando 7 como muito fácil. No meio do tempo estipulado, registramos a média de 8,8, classificando 9 como fácil. E, no final, marcamos a média de 8,7, considerando a percepção de esforço dos indivíduos como exercício moderado.

Os números de 6 a 20 são baseados na Frequência Cardíaca de 60-200bpm. Sendo que o número 12 corresponde a aproximadamente 55\% e o número 16 a 85\% da Frequência Cardíaca Máxima. Observando que os participantes permaneceram em nível normal de frequência cardíaca quando executaram o esforço, respaldando que o exercício proposto foi moderado.

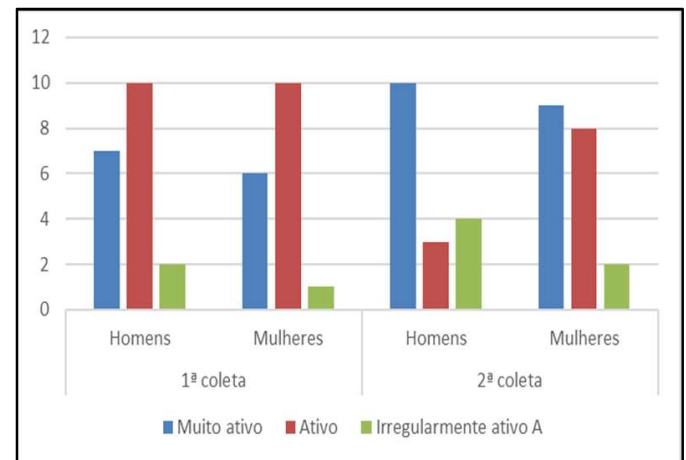

Gráfico 2: Avaliação do IPAQ nas coletas de fevereiro e julho, divididos em homens e mulheres. 
Ainda sobre os resultados referentes ao nível de atividade física, realizamos o teste Qui-Quadrado para verificar se realmente as fases das coletas de dados influenciavam no resultado do IPAQ. Os resultados demonstraram que a classificação do IPAQ independe da época da coleta ( $p$-valor 0,09). 0 gráfico 2 traz a avaliação do nível de atividade física nos dois momentos das fases divididos por sexos.

No gráfico 2, comparando os dois momentos, obtivemos valores equilibrados para ambos os sexos, mostrando um valor expressivo de participantes muito ativos. Em relação às fases da coleta, o sexo dos participantes não influenciou na classificação do IPAQ ( $p$-valor 0,8 na primeira fase e 0,2 na segunda fase). Verificamos que os entrevistados tiveram aumento para muito ativos na segunda fase. Na primeira fase, o valor de muito ativos para homens foi $36 \%$ ( 7 homens) e na segunda fase foi $58 \%$ ( 10 homens).

Na primeira fase, o valor de muito ativas para mulheres foi 35\% (6 mulheres) e na segunda fase foi 47\% (9 mulheres). Os sexos apresentam diferenças fisiológicas, porém, em nosso estudo, constatamos que ambos apresentaram semelhantes adaptações ao treinamento aeróbio, respeitando sua individualidade biológica, assim, tanto as mulheres quanto os homens se tornaram mais ativos na segunda fase. No Gráfico 3, apresentamos a média de respostas "sim" referente ao questionário de sintomas respiratórios para a primeira e segunda fases.

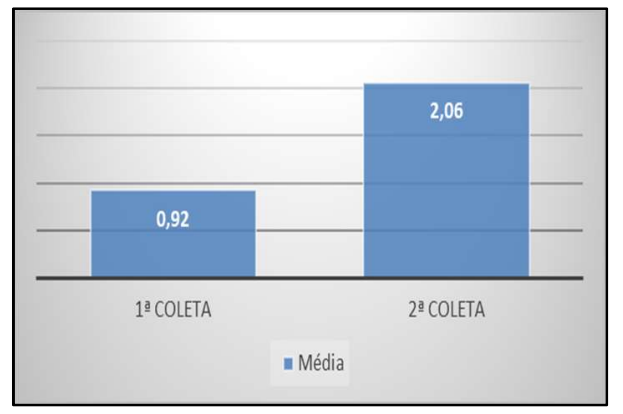

Gráfico 3: Média de respostas "sim" do questionário de sintomas respiratórios.

Os resultados apresentados no Gráfico 4 mostram que houve aumento de $123 \%$ na segunda fase em relação à pontuação do questionário referente às queixas respiratórias, tais como o reflexo do espirro, do pigarrear e da tosse, que expulsam as partículas invasoras, evitando as infecções. Podemos reforçar que o aumento de queixas na segunda fase se deu em razão de um aumento das taxas de poluição atmosférica e sua correlação com a diminuição das taxas pluviométricas do período estudado (jul/2016). No gráfico 4, apresentamos a média de respostas SIM (o sim de cada resposta, evidencia maiores queixas respiratórias, de acordo com o questionário aplicado) em homens e mulheres do Questionário de Sintomas respiratórios da primeira e segunda fases.

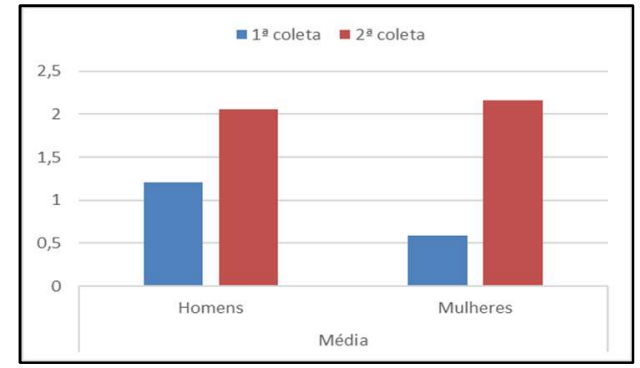

Gráfico 4: Média de respostas SIM de homens e mulheres do Questionário de Sintomas respiratórios da 1a e 2a fases. 
Notamos que a média de respostas 'sim', trazida no gráfico 4 , obteve um aumento expressivo para ambos os sexos. Torna-se interessante esclarecermos os seguintes relatos de percepção respiratória, obtidos com o questionário de sintomas respiratórios. Entre as observações estão o reflexo do espirro, do pigarrear e da tosse, que expulsam as partículas invasoras, evitando as infecções (BRAGA, 2003).

Na primeira fase da pesquisa, os homens se queixaram de alergias (31\%), tosse $(26 \%)$, sensação de aperto (21\%) e secreção nasal (15\%). Na segunda fase, verificamos aumento da percepção de alergias (52\%), tosse $(42 \%)$, e secreção nasal $(26 \%)$. Com relação às mulheres, na primeira fase elas se queixaram de sensação de aperto (11\%), tosse (11\%), alergias (11\%) e secreção nasal (6\%). Ao passo que, na segunda fase, registramos aumento mais expressivo de tosse $(82 \%)$, alergias (70\%), secreção nasal $(29 \%)$ e sensação de aperto (17\%).

Em seu estudo, Rondon (2011) identificou sintomas respiratórios como indicadores de estado de saúde em trabalhadores de instalações de fabricação de cerâmica apresentaram prevalência de queixas de 78\% para 'sintomas respiratórios gerais'. A tosse foi relatada como o sintoma mais prevalente nos trabalhadores expostos à poeira de sílica.

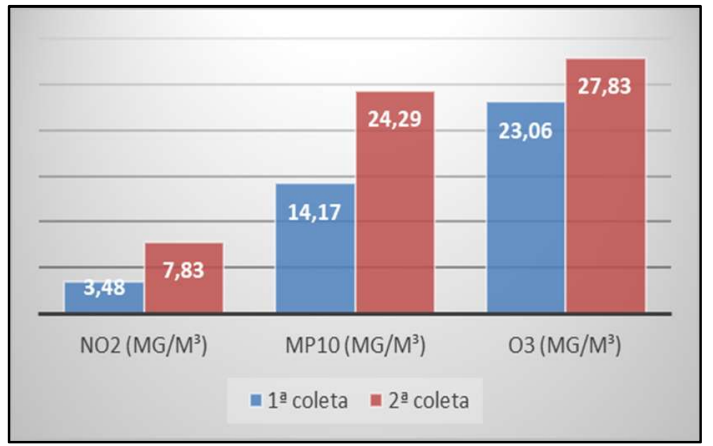

Gráfico 5: Média de concentração dos poluentes medidos pela CETESB. Legenda: $\mathrm{MP}_{10}$ - Material Particulado; $\mathrm{NO}_{2}$ - Dióxido de Nitrogênio; $\mathrm{O}_{3}$ - Ozônio.

Tais dados podem ser relacionados aos níveis de poluentes atmosféricos apresentados no Gráfico 6, cujas medições dos poluentes demonstraram que os níveis de poluentes atmosféricos, segundo a legislação e as resoluções do Conama, estão abaixo dos padrões primários e secundários de qualidade do ar, ou seja, as concentrações dos poluentes nesses pontos não ocasionariam problemas de saúde humana e nem provocariam danos à fauna, à flora, aos materiais e ao meio ambiente em geral. Entretanto, na segunda fase (jul/2016), verificamos que as concentrações de poluentes mensurados pela CETESB aumentaram, como podemos constatar por meio dos dados apresentados no gráfico 5.

Com base no Gráfico 5, notamos que houve um aumento da primeira para a segunda fase na concentração dos poluentes, o $\mathrm{NO}_{2}$ teve um aumento de $125 \%$, o $\mathrm{MP}_{10}$ teve um aumento de $100 \%$ e o $\mathrm{O}_{3}$ teve um aumento de $20 \%$. Contudo, é importante entender que apesar dos poluentes atmosféricos estarem abaixo do nível do relatório de qualidade do ar, houve um aumento de $123 \%$ das queixas respiratórias da primeira para a segunda fase (Gráfico 3), relacionado ao aumento de poluentes (Gráfico 4).

O clima é evidenciado pelas anomalias em seus elementos (temperatura, umidade relativa do ar, direção e velocidade do vento), sobretudo quando o processo de urbanização carece de planejamento 
adequado ao ambiente em que a cidade está inserida. Como afirma Amorim (2000), as associações dos elementos do relevo e a arborização com as diferentes ocupações do solo, especialmente no que se refere às construções e às circulações de veículos e pessoas, nos ambientes interurbanos, podem originar peculiaridades em suas características térmicas e higrométricas. As variáveis de velocidade do vento, temperatura e umidade relativa do ar, que analisamos neste estudo, estão representadas no gráfico 6, nas duas fases de coleta.

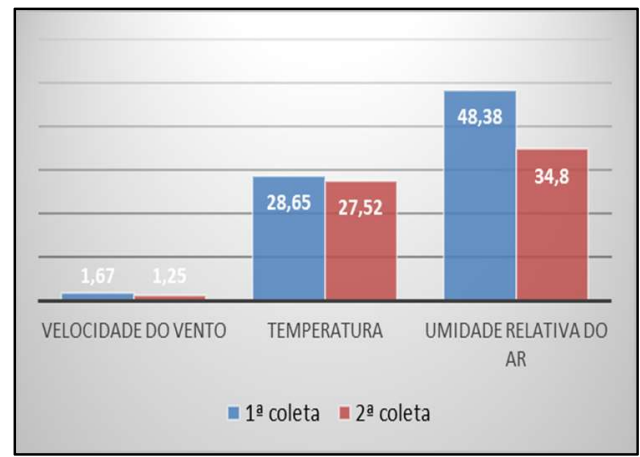

Gráfico 6: Médias das variáveis (velocidade do vento, temperatura, umidade) das 1ำ e 2으 fases.

O resultado apresentado no gráfico 6 mostra que a umidade relativa do ar diminuiu (28\%) de forma expressiva na segunda fase. Ao passo que as outras variáveis (velocidade do vento e temperatura) não apresentaram diferença expressiva entre as duas coletas. A baixa umidade do ar provoca uma série de incômodos, além de ressecar a pele, favorece o surgimento de doenças respiratórias. A esse respeito, Braga (2003) acrescenta que a baixa umidade resseca as vias aéreas e compromete a proteção natural do nariz, que é feita por uma secreção líquida que reveste a região, facilitando a entrada de vírus e bactérias que já se proliferam e deixam o corpo mais vulnerável.

O tempo seco também dificulta a dispersão de poluentes, desde a poeira até a poluição que sai dos automóveis, passando por ácaros e fungos, ficam suspensos no ar e podem ser inalados, o que favorece problemas respiratórios e infecções (BRAGA, 2003). A baixa umidade do ar, quando relacionada à poluição, pode causar malefícios à saúde, tais como ressecamento dos olhos, nariz e garganta, sendo essa uma possível causa do aumento de queixas respiratórias indicadas no presente estudo (Gráfico 4). Da mesma forma, na segunda fase, identificamos o aumento dos poluentes atmosféricos (Gráfico 5).

A interação entre os gases poluentes e a atmosfera define o grau de qualidade do ar. Isto é, quanto maior a concentração de um ou mais poluentes na atmosfera, pior será a qualidade do ar. Logo, considerando uma fonte emissora contínua, dependerá das características da atmosfera em dissipar ou concentrar mais a poluição (FERREIRA, 2016). Presidente Prudente localiza-se sob um regime de clima tropical, numa área de transição climática, sofrendo a atuação da maioria dos sistemas atmosféricos presentes na América do Sul. Enquanto os sistemas tropicais lhe conferem elevadas temperaturas de primavera e verão, os sistemas extratropicais ocasionam episódios de invasão das frentes frias e do ar polar no outono e inverno, provocando baixas temperaturas (SANT'ANNA NETO et al., 2009). 


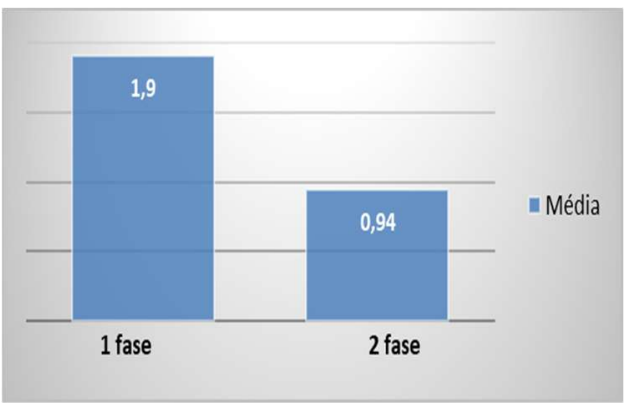

Gráfico 7: Média do COex (imediatamente após exercício) para as 1으 e a 2으 fases.

Segundo dados obtidos junto ao COex, houve diminuição de 50,5\% do monóxido de carbono ar exalado na segunda fase, como mostram os resultados expressos no gráfico 7, referentes aos participantes imediatamente após o exercício. Este resultado confirma a relação da eficiência cardiovascular e pulmonar com a prática de exercícios físicos. O sistema cardiovascular e o pulmonar estão diretamente ligados à prática do treinamento aeróbio, pois é muito útil em exercícios prolongados com maior eficiência ventilatória, significando mais oxigênio para os músculos ativos.

Varas (2009) salientou que a absorção de CO é diretamente proporcional à concentração do ambiente, ao tempo de exposição e à velocidade de ventilação, que também dependerá da atividade durante o tempo de exposição, sendo igualmente observado em outros estudos. Nos achados de Santos (2001) a eliminação de COex é aumentada pela realização de exercícios.

Os dados nos mostraram que os voluntários se tornaram mais ativos na segunda fase da pesquisa, isto possibilitou maior ventilação pulmonar, por meio da atividade moderada, melhorando suas respostas fisiológicas e reduzindo os dados do monóxido de carbono no ar exalado imediatamente após o exercício, promovendo, também, uma diminuição da absorção do monóxido de carbono no ar exalado, mostrando que em indivíduos sadios o organismo dá resposta imediata das perturbações externas, no caso a poluição atmosférica. Verificamos que a prática regular e contínua de exercícios físicos aeróbios é capaz de amenizar os danos fisiológicos no organismo humano causados pela exposição a poluição atmosférica.

Guyton et al. (1998) afirma que maior ventilação pulmonar, por meio da atividade moderada, melhora a resposta fisiológica reduzindo os índices do monóxido de carbono no ar exalado imediatamente após o exercício. Em seu estudo, Cuissi (2014) corrobora com este resultado, quando afirma que em indivíduos saudáveis o sistema de defesa encontra-se íntegro, desempenhando sua função de eliminar os agentes nocivos $\mathrm{NO}_{2}$ e o $\mathrm{CO}$.

Ressaltando essas adaptações no sistema respiratório, Braga (2003) reitera que os mecanismos de defesa, como a filtração aerodinâmica e o transporte mucociliar, colaboram com a eliminação das partículas de poluentes. Estes resultados também são apresentados no estudo de Pinho (2006) mostrando evidências de que o exercício físico regular em esteira pode ser um agente capaz de amenizar os danos oxidativos pulmonares induzidos pela inalação de partículas de carvão mineral.

Podemos descrever essa diminuição como quebra da homeostase corporal, pois o corpo humano, ao entrar em contato com o exercício físico, recebe estímulos fisiológicos desregulando seu equilíbrio de base, 
buscando adaptações fisiológicas para o novo estímulo proposto, por meio da supercompensação do organismo (MIRANDA et al., 2008).

Apesar das respostas fisiológicas ao exercício ser semelhante em mulheres e homens, verificamos algumas diferenças a serem consideradas. A mais marcante envolvendo mulheres consistiu no maior consumo de oxigênio durante a caminhada ou corrida de intensidade submáxima (menor eficiência mecânica), o que acarreta menor reserva metabólica e fadiga precoce (ACSM, 2010). A relação entre a resposta fisiológica diferente entre homem e mulher pode acarretar na resposta diferente entre o ar exalado. Durante exercícios aeróbios ocorre menor consumo máximo de oxigênio em mulheres em comparação com os homens, e o principal mecanismo hemodinâmico envolvido é o menor débito cardíaco decorrente de menor volume sistólico.

\section{CONSIDERAÇÕES FINAIS}

O estudo permitiu constatar que o exercício físico provoca uma série de respostas fisiológicas, resultantes de adaptações autonômicas e hemodinâmicas que influenciarão o sistema cardiovascular e representam importante papel como elemento não medicamentoso para o seu controle ou como adjuvante ao tratamento farmacológico.

O presente estudo mostrou que os participantes da pesquisa se tornaram mais ativos, sendo possivelmente estimulados e motivados pelo ambiente com grande presença de vegetação e boa infraestrutura do local. Importante ressaltarmos que este estudo também ofereceu aos participantes orientações dirigidas, como educação em saúde, propiciando-lhes incentivo para uma melhor frequência e maior intensidade, fato que pudemos observar na segunda coleta de dados, em que o número de praticantes mais ativos apresentou um aumento.

A investigação da interdependência da atividade física, saúde e meio ambiente se resulta num elemento essencial na sociedade, permitindo, assim, recomendar medidas eficazes para a saúde coletiva, colaborando com o bem-estar de todos. Podemos ressaltar que houve um aumento nas queixas respiratórias -tosse, alergias e secreções nasais - entre os participantes na segunda coleta, que pode ter sido causada pela associação da poluição atmosférica com a baixa umidade relativa do ar caracterizada no momento da segunda fase.

As correlações entre o COex e os poluentes atmosféricos apresentaram correlações sem discrepância (fracas e não significativas). O estudo se justifica por apontar a importância da prática do exercício físico, mesmo que seja em ambiente aberto, e mostra que quanto maior a frequência e as intensidades desta prática, melhor a capacidade de adaptações fisiológicas do organismo à poluição.

\section{REFERÊNCIAS}

ACSM. American College of Sports Medicine. ACSM's Guidelines for Exercise Testing and Prescription. 8 ed. Philadelphia: ACSM, 2010.
AMORIM, M. C. C. T.. O clima urbano de Presidente Prudente/SP. Tese (Doutorado em Geografia) -Universidade de São Paulo, São Paulo, 2000. 
ANDRADE, J. P.. VI Diretrizes Brasileiras de Hipertensão. Arquivos Brasileiros de Cardiologia, Rio de Janeiro v.95, n.1, p.1-51, 2010.

ARANA, A. R. A.; XAVIER, F. B.. Qualidade ambiental e promoção de saúde: um estudo sobre o Parque do Povo de Presidente Prudente - SP. Revista do Departamento de Geografia, São Paulo, v.32, p.1-14, 2016.

ARBEX, M. A.. A poluição do ar e o sistema respiratório. Jornal Brasileiro de Pneumologia, São Paulo, v.38, p.643655, 2012.

AUBERT, A. E.; BECKERS, F.; RAMAEKERS, D.. Short-term heart rate variability in young athletes. Journal of Cardiology, n.37, p.85-88, 2001.

BENINI, S. M.. Áreas verdes públicas: a construção do conceito e a análise geográfica desses espaços no ambiente urbano. Dissertação (Mestrado em Geografia) Universidade Estadual Paulista, Presidente Prudente, 2009.

BORG, G. A. V.. Psychophysical bases of perceived exertion. Medicine \& Science in Sports \& Exercise, v.14, n.5, p.377381, 1982.

BORTOLO, C. A.. O espaço público do Parque do Povo: Presidente Prudente - SP: Reflexões geográficas. GeoAtos, Presidente Prudente, v.1, n.13, p.50-65, 2013.

BRAGA, A.. Poluição atmosférica e seus efeitos na saúde humana. São Paulo: USP, 2003.

BRILHANTE, O. M.; CALDAS, L. Q. A.. Gestão e avaliação de risco em saúde ambiental. Rio de Janeiro: FIOCRUZ, 1999.

CANÇADO J. E. D.. Repercussões clínicas da exposição à poluição atmosférica. Jornal Brasileiro de Pneumologia, São Paulo, v.32, n.2, p.5-11, 2006

CASTRO, H. A.; GOUVEIA, N.; ESCAMILLA-CEJUDO, J. A.. Questões metodológicas para a investigação dos efeitos da poluição do ar na saúde. Revista Brasileira de Epidemiologia, São Paulo, v.6, n.2, p.135-149, 2003.

CETESB. Companhia de Tecnologia de Saneamento Ambiental. Relatório de qualidade do ar no estado de São Paulo. São Paulo: CETESB, 2014.

CONAMA. Conselho Nacional do Meio Ambiente. Resolução n.01 de 23 de janeiro de 1986. Brasília: CONAMA, 1986.

COSTA, A. E.. Parque do Povo: Tribos Urbanas e Territórios. Monografia (Graduação em Geografia) - Universidade Estadual Paulista, Presidente Prudente, 2009.

CUISSI, R. C.. Efeitos da poluição atmosférica no sistema respiratório de indivíduos praticantes de exercício físico aeróbio em ambiente aberto e fechado. Dissertação (Mestrado em Fisioterapia) -Universidade Estadual Paulista, Presidente Prudente, 2014.

DENATRAN. Departamento Nacional de Trânsito. Frota de veículos: 2000 a 2014. Brasília: DENATRAN, 2014.

DICKEY, J. H. Part VII. Air pollution: overview of sources and health effects. Disease a Month, v. 46, p. 566-589, 2000.
ESTEVES, G. R. T. Estimativa dos efeitos da Poluição Atmosférica sobre a Saúde Humana: algumas possibilidades metodológicas e teóricas para a cidade de São Paulo. In: ENCONTRO DA ASSOCIAÇÃO NACIONAL DE PÓSGRADUAÇÃO E PESQUISA EM AMBIENTE E SOCIEDADE, 2. Anais. Indaiatuba: ANPPAS, 2004.

FERREIRA, C. C. M.; OLIVEIRA, D. E.. Estimativa da poluição veicular e qualidade do ar nas principais vias do sistema viário da região central da cidade de Juiz de Fora. Revista do Departamento de Geografia, São Paulo, p.98-114, 2016.

FISHMAN, A. P. E. J.A.; FISHMAN, J.A.; GRIPPI, M.A.; SENIOR, R.M.; PACK. A.. Fishman's pulmonary diseases and disorders. Philadelphia: McGraw-Hill Professional, 2008.

GUYTON, A. C.; HALL, J. E.. Fisiologia Humana e Mecanismos das Doenças. Rio de Janeiro: Guanabara Koogan, 1998.

LASTE, R.. Exercício físico e poluição atmosférica: ambiente externo vs interno. Revista Educação, Meio Ambiente e Saúde, Manhuaçu, v.4, n.1, p.51-64, 2009.

MIOT, H. A.. Tamanho da amostra em estudos clínicos e experimentais. Jornal Vascular Brasileiro, São Paulo, v.10, n.4, p.275-278, 2011.

MIRANDA, M. J.; BAPTISTA, T. J. R.. A Poluição do ar na cidade de Goiânia - GO e a prática de exercícios físicos. Educação Física em Revista, Goiânia, v.2, n.3, 2008.

MOTA, S.. Urbanização e Meio Ambiente. 3 ed. Rio de Janeiro: ABES, 2003

OLIVEIRA, L. A.; MASCARÓ, J. J.. Análise da qualidade de vida urbana sob a ótica dos espaços públicos de lazer. Ambiente Construído, Porto Alegre, v.7, n.2, p.59-69, 2007.

OLIVEIRA, R. C.. Efeito da composição do MPfino, PM2,5 e "Residual Oil Fly Ash" (ROFA), como determinante do potencial mutagênico e tóxico celular: um estudo em bioensaios vegetais e animais. Tese (Doutorado em Ciências) - Universidade de São Paulo, São Paulo, 2006.

PELICIONI, M. C. F.. Educação Ambiental: evolução e conceitos. In: PHILIPPI JUNIOR, A.. Saneamento, saúde e ambiente: fundamentos para um desenvolvimento sustentável. Barueri: Manole, 2005. p.587-598.

PINHO, R. A.. Exercício físico regular diminui o estresse oxidativo pulmonar em ratos após exposição aguda ao carvão mineral. Revista Brasileira de Medicina do Esporte, Criciúma, v.12, n.2, 2006.

RIBEIRO, H.; ASSUNÇÃO, J. V.. Efeitos das queimadas na saúde humana. Estudos Avançados, São Paulo, v.16, n.44, p.125-148, 2002.

SALICIO, M. A.. Environmental variables and levels of exhaled carbon monoxide and carboxyhemoglobin in elderly people taking exercise. Ciência \& Saúde Coletiva, Cuiabá, v.21, n.4, p.1023-1032, 2016.

SANCHES, R. A.. Avaliação de impacto ambiental e as normas de gestão ambiental da série ISO 14000: características técnicas e subsídios à integração. Dissertação 
(Mestrado em Ciências da Engenharia Ambiental) -

Universidade de São Paulo, São Carlos, 2011.

SANT'ANNA NETO, J. L.; TOMMASELLI, J. T. G.. O tempo e o clima de Presidente Prudente. Presidente Prudente: UNESP, 2009.

SANTOS, U. P.. Emprego da determinação de monóxido de carbono no ar exalado para a detecção do consumo de tabaco. Jornal Brasileiro de Pneumologia, São Paulo, v.27, n.5, p.213-236, 2001

SAWADA, F. T.. Análise espaço-temporal do parque do povo de Presidente Prudente a partir de fotos aéreas e imagem orbital. Monografia (Graduação em Engenharia Cartográfica) - Universidade Estadual Paulista, Presidente Prudente, 2007.

SILVA, M. J. M.. O parque do povo em Presidente Prudente: a lógica da intervenção do poder público na (re)estruturação do espaço urbano. Dissertação (Mestrado em Geografia) Universidade Estadual Paulista, Presidente Prudente, 1994.

TEIXEIRA, J. A. C. Hipertensão arterial sistêmica e atividade física. Revista da Sociedade de Cardiologia do Estado do Rio de Janeiro, Niterói, v.13, p.25-30, 2000.

VARAS, H.. Prevención de Accidentes por Inhalación de Monóxido de Carbono em Edificios. Uma Intervención Comunitaria. Revista Ciência e Trabalho, Santiago, v.11, n.31, p.25-31, 2009.

VAZ, R.. As precipitações extremas e inundações em áreas de fundos de vale em Presidente Prudente: um estudo sobre o parque do povo e Prudenshopping. Monografia (Graduação em Geografia) - Universidade Estadual Paulista, Presidente Prudente, 1999. 